\title{
Possible photohadronic origin of the IC-201114A alert
}

\author{
Alberto Rosales de León, ${ }^{a, *}$ Anthony M. Brown ${ }^{a}$ and Paula M. Chadwick ${ }^{a}$ \\ ${ }^{a}$ Centre for Advanced Instrumentation (CfAI), Department of Physics, University of Durham, \\ South Road, Durham DH1 3LE, UK. \\ E-mail: alberto.rosales-de-leon@durham.ac.uk
}

The Icecube neutrino observatory is a cubic-kilometer particle detector located at the South Pole. A system of public real-time alerts on neutrino candidate events likely to be of astrophysical origin has been operating since 2016. On November 14th 2020, a track-like event with a high probability of being of astrophysical origin (IC-201114A) was reported. 4FGL J0658.6+0636, a source of the blazar type, was identified inside the $90 \%$ localisation region of the alert $0.8^{\circ}$ from the best-fit event position by the Fermi-LAT collaboration. In this work, we analyse 12.3 years of Fermi-LAT data from 4FGL J0658.6+0636. No indication of significant gamma-ray activity was found around the time of the alert, however, two periods in which the source was detected significantly were identified and studied considering a lepto-hadronic scenario. We investigate a possible photohadronic origin for high energy neutrinos and calculate the gamma-ray contribution to the spectral energy distribution (SED). The predicted neutrino flux and the expected time for a neutrino detection from the source during a flaring state were calculated for the periods of significant activity. Assuming the historical behaviour of the source, an approximation of the gamma-ray and neutrino flux coming from photohadronic interactions around the IC-alert is also given.

$37^{\text {th }}$ International Cosmic Ray Conference (ICRC 2021)

July 12th - 23rd, 2021

Online - Berlin, Germany

\footnotetext{
*Presenter
} 
Alberto Rosales de León

\section{Introduction}

Recent discoveries have propelled multi-messenger astronomy to the forefront of astrophysics. The information provided by the combined observations of two or more cosmic messengers is a powerful tool to identify and understand some of the most energetic phenomena in the universe.

Blazars are a sub-class of radio-loud Active Galactic Nuclei (AGN) with a relativistic jet pointing close to our line of sight. These objects undergo flaring states, periods of enhanced activity on time scales that can last minutes to months. In the standard leptonic scenario, the typical Spectral Energy Distribution (SED) shape is interpreted as the combination of two processes: synchrotron and synchrotron-self Compton (SSC) radiation. However, it has been suggested that AGN might be astrophysical spots of cosmic-ray acceleration, which means that high energy neutrinos alongside gamma-rays might be produced via hadronic interactions, protons can interact either with photon target fields ( $p \gamma$ interactions) or dust clouds of surrounding material ( $p p$ interactions).

Recentely the IceCube collaboration released a 10 year data sample of track-like neutrino candidates [1], which showed evidence at the $3.3 \sigma$ level with respect to the atmospheric neutrino background of a cumulative excess of events coming principally from the Seyfert II galaxy NGC 1068, and 3 blazars: TXS 0506+056, PKS 1424+240 and GB6 J1542+6129.

In 2016, IceCube initiated a system of public real-time alerts on events likely to be of astrophysical origin [2]. The reconstructed direction of these events can be used to perform follow-up multiwavelength (MWL) observations (radio, infrared, visible, X-ray, gamma-ray) and identify possible electromagnetic counterparts. On September 22nd 2017, an extensive multiwavelength (MWL) campaign was triggered after the detection the IC-170922A alert. The blazar TXS 0506+056, located at just $0.1^{\circ}$ from the best-fitting neutrino direction was reported to be in a flaring state by the Fermi-LAT Collaboration [3]. This case represented the first plausible association of a high energy neutrino alert with an astrophysical point source. In addition, a time-dependent analysis done by the IceCube collaboration found an excess of $13 \pm 5$ high energy neutrino events coming from the direction of this source between September 2014 and March 2015 [4]. The latter analysis represents $\mathrm{a} \sim 3.5 \sigma$ evidence for neutrino emission from the direction of TXS 0506+056, and stands as an independent result from the 2017 observations.

\section{IC-201114A neutrino alert}

The IceCube collaboration reported a track-like event on November 14th 2020 at 15:05:31.96 UT (T0=59167.6288 MJD). This event was classified with a high probability of being from astrophysical origin (0.562) and a false alarm rate of 0.92 events per year due to the atmospheric neutrino background. The refined best fit position (statistical uncertainty only) was RA $=105.25^{\circ}$ $+1.28^{\circ} / 1.12^{\circ}, \operatorname{Dec}=6.05^{\circ} \pm 0.95^{\circ}(\mathrm{J} 2000)$ for the $90 \%$ containment region. The most likely neutrino energy was set at $214.29 \mathrm{TeV}$ [5]. A source of the blazar type was identified by the Fermi-LAT collaboration inside the $90 \%$ localisation region of IC-201114A, at a distance of $0.81^{\circ}$ from the best-fit event position: 4FGL J0658.6+0636 (also known as NVSS J065844+063711), located at $\mathrm{RA}=104.64^{\circ}$, Dec $=6.60^{\circ}$ (J2000) [6]. This source has been recently classified as a BL Lac object with a high-synchrotron peak (HSP; $v>10^{15} \mathrm{~Hz}$ ) and a lower limit in redshift of $z>0.5$ [7]. It was reported no significant $(>5 \sigma)$ gamma-ray activity from the source in a preliminary analysis of 
Fermi-LAT data on 1-day and 1-month timescales prior to the neutrino alert. However, an analysis of more than 12-years of LAT data (2008-08-04 to 2020-11-14 UTC) identified this object as a very high energy (VHE; E $>20 \mathrm{GeV}$ ) source [8].

\section{Photohadronic Interactions}

To investigate the possibility of photohadronic contributions to the SED of 4FGL J0658.6+0636, a standard leptonic interpretation is assumed to contribute to the SED. It is considered that SSC photons will provide a target photon field for $p \gamma$ interactions to arise inside a single spherical emission region (blob) in the blazar's jet. Typical value parameters were assumed for the modelling (see Table 1). We use the $\Delta^{+}$-resonance approximation for the $p \gamma$ interactions [9]. At its peak $\left(m_{\Delta}=1.232 \mathrm{GeV}\right)$, the cross-section of the $\Delta^{+}$-resonance reaches a value of $\sigma_{\text {peak }} \sim 500 \mu$ barn $=$ $5 \times 10^{-28} \mathrm{~cm}^{2}$, this is a factor of $\sim 5$ times larger than the cross section from the direct channel production. The photohadronic scenario, has been applied previously to the blazar Markarian 421 during a prominent flare in 2010 [10]; our results showed the potential of including $p \gamma$ interactions in blazar modelling.

The gamma-ray component from the photohadronic component will depend proportionally on the background target photons $\left(\Phi_{\text {input }}\right)$ and the injected proton spectrum as:

$$
F_{\gamma}\left(E_{\gamma}\right)=A_{\gamma} \Phi_{\text {input }}\left(\epsilon_{\gamma}\right)\left(\frac{E_{\gamma}}{\mathrm{TeV}}\right)^{-\alpha+3} \mathrm{e}^{-\tau_{\gamma \gamma}\left(E_{\gamma}, \mathrm{z}\right)} .
$$

where $A_{\gamma}$ is a dimensionless normalisation constant that groups together a series of physical parameters and proportionality constants, while $\Phi_{\text {input }}\left(\epsilon_{\gamma}\right)$ is approximated from a Fermi-LAT analysis to the source. The attenuation effect produced by the Extragalactic Background Light (EBL) is taken into account with the model of Dominguez et al. [11], where $\tau_{\gamma \gamma}$ is the optical depth.

The neutrino spectrum is assumed be a PL $\frac{\mathrm{d} N}{\mathrm{~d} E_{v}}=A_{v} E_{v}^{-\beta}$; where $A_{v}$ is the neutrino flux normalisation constant and a power index of $\beta=2$ is assumed. The gamma-ray flux and the neutrino flux have a common origin and can be related as described in Halzen and Hooper (2005) [12]. The expected number of neutrino-induced muon events can be approximated using the effective area from the operational IceCube configuration:

$$
N_{\text {events }}=T \int_{E_{v, \text { min }}}^{E_{v, \text { max }}} \frac{\mathrm{d} N}{\mathrm{~d} E_{v}} A_{\mathrm{eff}}\left(E_{v}, \delta\right) \mathrm{d} E_{v}
$$

where $A_{\text {eff }}$ is the effective area of the IceCube configuration under consideration, and $\mathrm{T}$ is the observation length. The numerical values used for the different IceCube configurations (IC40, IC59, IC79, IC86) are available from the IceCube data releases web-page ${ }^{1}$.

Most of the events detected with IceCube come from the atmospheric background, muons or neutrinos that are created when cosmic-rays interact with the atmosphere. According to the IceCube collaboration, a high-significance point source detection can require as few as two or three, or as many as 30 [4], signal events to stand out from the background, depending on the energy

\footnotetext{
${ }^{1}$ See https://icecube.wisc.edu/science/data-releases/
} 


\begin{tabular}{cc}
\hline Parameter & Typical Value / Range \\
\hline$z$ & $0.5-1.5[7]$ \\
$R_{f}^{\prime}$ & $(1.35-2.25) \times 10^{16} \mathrm{~cm}^{2}$ \\
$B$ & $1 \mathrm{G}[13]$ \\
$\mathcal{D}$ & $13[14]$ \\
$\alpha$ & $2.2[15]$ \\
\hline$E_{p}$ & $663 \mathrm{GeV}-47 \mathrm{PeV}$ \\
$E_{v}$ & $30 \mathrm{GeV}-2.35 \mathrm{PeV}$ \\
\hline
\end{tabular}

Table 1: Summary table of the parameters used to characterise the emission region of 4FGL J0658.6+0636; typical values for blazars were assumed. The energy ranges for the accelerated protons and neutrinos produced were calculated using the redshift range in the observer's reference frame.

spectrum and the clustering of events in time. We assume that at least two neutrino events above the background level are required for a high-significance point source detection, and define the minimum detection time (MDT) as the estimated time elapsed for IceCube to detect this minimum quota during an active state of the source, but it is worth mentioning that a higher number of events might be needed for a positive detection.

\section{Gamma-ray activity}

We performed two Fermi-LAT analyses of 4FGL J0658.6+0636: one using a 12.3-year data set (54683-59178 MJD) to characterise the historical gamma-ray flux and one concentrating on the 4-months around T0 of the neutrino alert (59108-59228 MJD). In both cases, the Pass8 version of the Instrument Response Functions (IRF)s with the 1.2.1 Fermi Science Tools were used alongside Fermipy 0.17.4 [16] and the 4FGL-DR2 catalog [17].

For the analyses, we considered a $15^{\circ}$ radius of interest (RoI) around the source position and a photon energy range between $200 \mathrm{MeV}$ and $300 \mathrm{GeV}$. A $90^{\circ}$ zenith cut angle was applied to avoid gamma-rays coming from Earth's atmosphere. The data were binned using 4 logarithmic bins per decade in energy and a spatial bin size of $0.1^{\circ}$ per pixel. 4FGL J0658.6+0636 is listed as a source with a Power-law (PL) spectrum: $\frac{\mathrm{d} N}{\mathrm{~d} \epsilon_{\gamma}}=N_{\mathrm{PL}}\left(\frac{\epsilon_{\gamma}}{\epsilon_{0}}\right)^{-\kappa}$; where $N_{\mathrm{PL}}$ represents a normalisation constant, $\kappa$ is the spectral index associated with the source and $\epsilon_{0}$ is the pivot energy in MeV. The input seed photon spectrum for the photohadronic model can be expressed as $\Phi_{\text {input }}=\epsilon_{\gamma}^{2} \frac{\mathrm{d} N}{\mathrm{~d} \epsilon_{\gamma}}$. The Galactic component was particularly significant going below $200 \mathrm{MeV}$ photon energy, so this is used as a lower energy limit in our analyses.

\subsection{3-year data set}

This analysis used a data set between 2008-08-04 and 2020-11-25 (54683 - 59178 MJD). 4FGL J0658.6+0636 was detected significantly with a TS=115 using the settings described above. A light curve for the 12.3-years of data was computed, the whole time period was divided into 10 evenly spaced bins. The bins identified as significant are shown in Figure 1; these are labelled and referred to from now on as BIN-A and BIN-B. For the bins during which there was no significant detection of the source, 95\% confidence level (CL) flux upper limits are shown. The average flux 


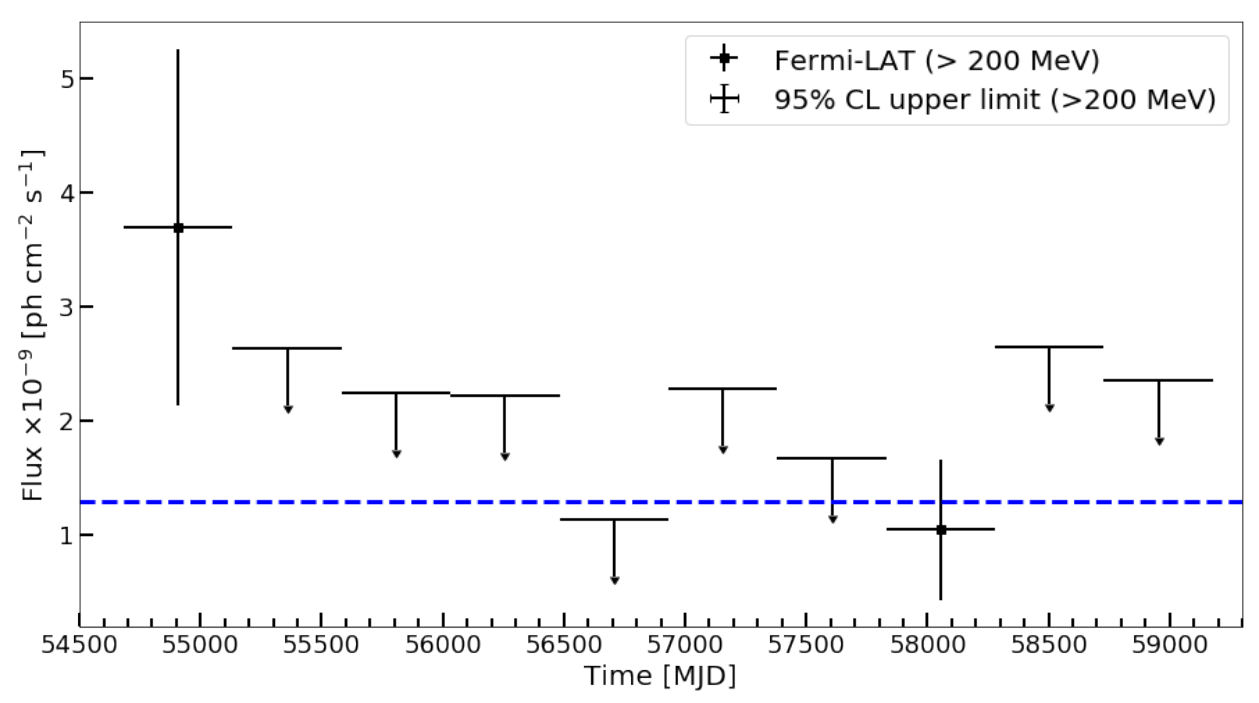

Figure 1: 12.3-year data set light curve of 4FGL J0658.6+0636, 10 evenly-spaced time bins were used in an energy range of $200 \mathrm{MeV}$ to $300 \mathrm{GeV}$. Significant bins (TS>25) are shown as square points, otherwise $95 \%$ CL upper limits appear. The horizontal blue line corresponds to the average flux of the source, $1.29 \times 10^{-9}$ $\mathrm{ph} \mathrm{cm}^{-2} \mathrm{~s}^{-1}$.

for the whole time period is $1.29 \times 10^{-9} \mathrm{ph} \mathrm{cm}^{-2} \mathrm{~s}^{-1}$, shown as a horizontal blue dotted line. The spectral parameters and flux of the significant bins are summarised in Table 2 alongside other model parameters used in the photohadronic fit.

BIN-A covers the period between 2008-08-04 to 2009-10-28, the source was detected in gamma-rays with $\mathrm{TS}=38$. The best-fit description is a power law with index $\kappa=2.1 \pm 0.2$ and a normalisation constant of $N_{\mathrm{PL}}=(8.9 \pm 2.2) \times 10^{-14} \mathrm{MeV} \mathrm{cm}^{-2} \mathrm{~s}^{-1}$. For BIN-A the predicted photohadronic fit does not match the Fermi-LAT spectrum.

Covering a time interval between 2017-03-17 and 2018-06-10, BIN-B was detected in gammarays with a TS $=34$. The spectral index calculated is $\kappa=1.5 \pm 0.2$ and $N_{\mathrm{PL}}=(4.6 \pm 2.1) \times 10^{-14}$ $\mathrm{MeV} \mathrm{cm}{ }^{-2} \mathrm{~s}^{-1}$. The Fermi-LAT SED for BIN-B is presented in Figure 2 (a). The pivot energy used was $\epsilon_{0}=2.75 \times 10^{3} \mathrm{MeV}$. The VHE emission (E>20 GeV) of 4FGL J0658.6+0636 was also studied. Over the 12.3-year period, the source was detected with a TS=34 in the VHE energy range. A VHE photon of $\sim 155 \mathrm{GeV}$ reported by [8] was found within the time range of BIN-B (59094.32 $\mathrm{MJD}$ ) and was the highest energy photon detected. The photohadronic contribution for this bin is shown in Figure 2 (a).

\subsection{4-month data set}

We searched for gamma-ray emission within a 4-month time window centred on T0, i.e. between 2020-09-16 (59108 MJD) and 2021-01-14 (59228 MJD). The specifications for the RoI model (size, energy range, spatial and energy binning, IRFs used) and optimisation steps followed were the same as described earlier. The source 4FGL J0658.6+0636 was not significantly detected $(\mathrm{TS}=3$ ) over the 4-month interval and mainly upper limits on the gamma-ray spectrum were obtained (see Figure 2 (b)). Assuming the source has a gamma-ray spectrum similar to that in BIN-B, but a flux that is just below the Fermi-LAT upper limits derived from our analysis, an approximation for 


\begin{tabular}{ccccccccc}
\hline BIN & TS & $\begin{array}{c}\text { Flux } \\
10^{-9}\left[\mathrm{ph} \mathrm{cm}^{-2} \mathrm{~s}^{-1}\right]\end{array}$ & $\begin{array}{c}N_{\mathrm{PL}} \\
10^{-14}\left[\mathrm{MeV} \mathrm{cm}^{-2} \mathrm{~s}^{-1}\right]\end{array}$ & $\kappa$ & $\begin{array}{c}A_{\gamma} \\
10^{-2}\end{array}$ & $\begin{array}{c}A_{v} \\
10^{-12}\left[\mathrm{TeV} \mathrm{cm}^{-2} \mathrm{~s}^{-1}\right]\end{array}$ & $\begin{array}{c}\text { MDT } \\
\text { Time }[\text { days }]\end{array}$ & $\begin{array}{c}F_{v, \text { int }} \\
10^{-5}\left[\mathrm{TeV} \mathrm{cm}^{-2}\right]\end{array}$ \\
\hline BIN-A & 38 & $3.69 \pm 1.56$ & $8.9 \pm 2.2$ & $2.1 \pm 0.2$ & $1.85-1.92$ & $6.35-6.93$ & 160 (IC40) & $8.66-9.46$ \\
& & & & & & & 100 (IC59) & $5.54-6.05$ \\
BIN-B & 34 & $1.04 \pm 0.61$ & $4.6 \pm 2.1$ & $1.5 \pm 0.2$ & $29.9-53.9$ & $186.15-352.21$ & 2.5 (IC86) & $4.02-7.61$ \\
\hline
\end{tabular}

Table 2: Summary table of the bins in the 12.3-year data set during which 4FGL J0658.6+0636 was detected significantly. The minimum detection time (MDT) as defined in Section 3 is given in the 8th column.

the photohadronic contribution to the SED is shown in Figure 2 (b). Here, the Fermi-LAT upper limits are used to set a normalisation constant $A_{\gamma}$, which depends on the redshift.

The purpose of our analyses was to investigate, in the context of the photohadronic approximation, whether we can account for the observed VHE energy gamma-ray data and what the neutrino flux would be from that contribution. We consider whether the non-detection of neutrinos from 4FGL J0658.6+0636 before the IC-201114A event is compatible with the gamma-ray emission coming from the photohadronic contribution and whether the detection of neutrinos from the object is compatible with the non-detection in gamma-rays around the time of IC-201114A.

\section{Neutrino Emission}

Considering a neutrino index of $\beta=2$, the neutrino flux normalisation constant values were estimated in the range of $A_{v}=(1.86-3.52) \times 10^{-10} \mathrm{TeV} \mathrm{cm}^{-2} \mathrm{~s}^{-1}$. The IC86 configuration was operational for the whole time range of this bin, assuming $p \gamma$ interactions and the average calculated neutrino flux, two neutrino event detections are expected in $\sim 2.5$-days, which is the minimum detection time for the redshift range considered. The integrated neutrino flux during this time span is predicted to be around $F_{v \text {,int }}=(4.02-7.61) \times 10^{-5} \mathrm{TeV} \mathrm{cm}^{-2}$.

For comparison purposes, to emulate the 13 excess events detected during the 2014-2015 neutrino flare of TXS 0506+056 [3], a detection time of $\sim 16$-days would be needed. It is therefore possible, according to the photohadronic scenario adopted, that IceCube would have detected an excess neutrino events during BIN-B. This is a strong motivation for investigating possible clustering of neutrino events from the direction of 4FGL J0658.6+0636 in historical IceCube data.

Using the spectral description from BIN-B, we can approximate the corresponding neutrino flux around the neutrino alert. A normalisation constant of $A_{v}=(1.05-1.78) \times 10^{-5} \mathrm{TeV} \mathrm{cm}^{-2} \mathrm{~s}^{-1}$ was calculated with an integrated flux of $F_{\nu}$,int $=(3.79-6.41) \times 10^{-5} \mathrm{TeV} \mathrm{cm}^{-2}$. The estimated MDT is $\sim 1$-hours. The non-detection of high-energy gamma-ray emission from 4FGL J0658.6+0636 around the time of the IC-201114A event is compatible with the detection of a neutrino, assuming the photohadronic scenario.

\section{Discussion}

Looking at 12.3-years of Fermi-LAT historical data, 2 significant periods of gamma-ray activity were identified and characterised (BIN-A and BIN-B). It was found that a dominant photohadronic contribution is compatible with the SED behaviour of the source during BIN-B, which presents a hard gamma-ray spectrum $(\kappa=1.5 \pm 0.2)$. A set of VHE photons with a $>90 \%$ probability of being from the source were detected along the 12.3-years data set, confirming 4FGL J0658.6+0636 as a 

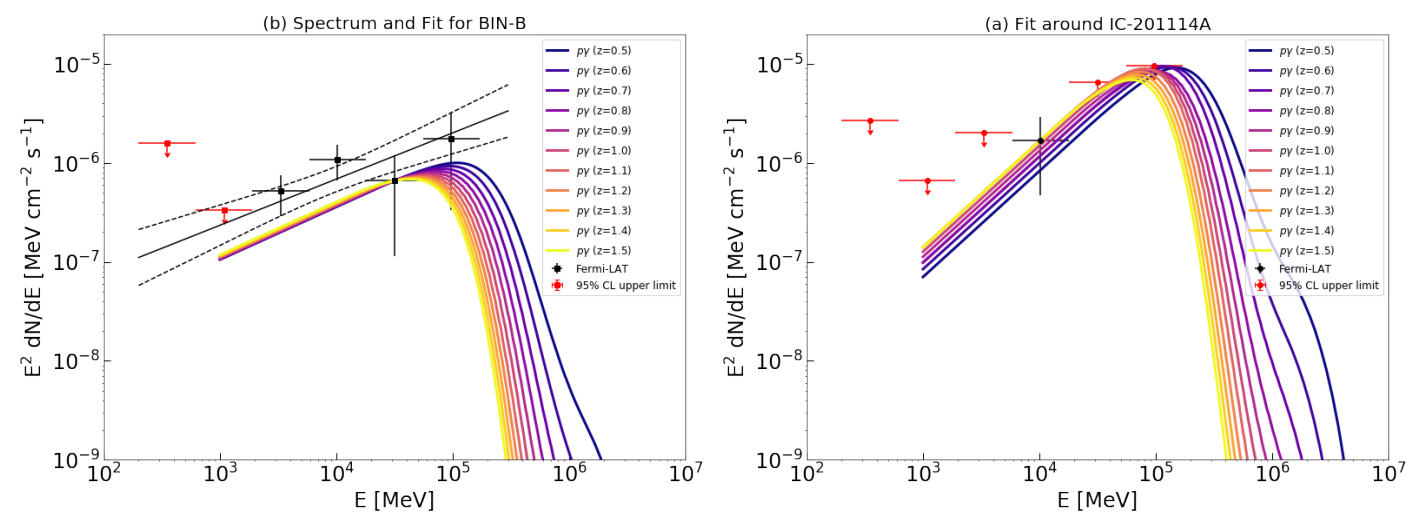

Figure 2: Fermi-LAT spectrum of 4FGL J0658.6+0636 and photohadronic fit obtained for: (a) BIN-B and (b) 4-month time window around IC-201114A. The calculated photohadronic contribution is plotted over a redshift range indicated by the colour scale.

VHE source. From this set, the highest energy photon detected was around $155 \pm 11 \mathrm{GeV}$ at 58146.30 MJD, which coincides with BIN-B (see Figure 1). From the estimated neutrino flux for this bin, a MDT of $\sim 2.5$-days is expected and at least 16-days to emulate the 13 excess events recorded during the 2014-2015 neutrino flare of TXS 0506+056. These results support the hypothesis that 4FGL J0658.6+0636 could be a neutrino emitter and, according to our predictions, it is possible that neutrinos from this astrophysical source could have been detected during the BIN-B time period.

In contrast, for BIN-A the predicted spectrum does not match the Fermi-LAT data points at energies below $100 \mathrm{GeV}$; this may indicate that leptonic components play an important role in the emission of VHE gamma-ray photons and are necessary to explain fully the emission during BIN-A.

In an optimistic scenario, our results predict a MDT of $\sim 1$-hours using the assumed conditions from BIN-B seed photon spectrum. These results are compatible with the non-detection in gammarays around the time of the neutrino detection but the main reason behind this phenomenon remains open to discussion. One option is to consider a high opacity given by a dense photon field inside the emission region. This condition could help to increase the efficiency of the $p \gamma$ interactions, but it also means that the internal absorption will diminish the observed gamma-ray flux through electromagnetic pair cascades. If the opacity is too large, then a possible neutrino detection will not be accompanied by an enhanced gamma-ray flux.

It is worth noticing that, with a reported signalness of 56\%, an origin for IC-201114A other than the blazar is not discarded. There is a chance of this being a high energy atmospheric event or part of the small diffuse neutrino flux contribution from the Galactic plane.

\section{Conclusions}

The blazar 4FGL J0658.6+0636 was recently identified as a possible counterpart for the IC201114A neutrino alert. Our analysis of 12.3-years of Fermi-LAT data confirmed this blazar as a VHE source. Two significant periods of gamma-ray activity were found (BIN-A and BIN-B) which were studied using a one-zone photohadronic scenario with $p \gamma$ interactions. 
It was found that the photohadronic approximation is compatible with the behaviour of the source during BIN-B. The gamma-ray spectrum during BIN-B was particularly hard, and coincided with the highest energy photon recorded from the source $(\mathrm{E}=155 \mathrm{GeV})$. Using this spectrum in the photohadronic model, and taking into account the IceCube sensitivity at the time, we calculated the minimum detection time to be MDT 2.5-days. The neutrino flux coming from the photohadronic approximation was estimated for a 4-month period around the IC-201114A neutrino alert. In an optimistic scenario, our results predicted a MDT between $~ 1$-hour with a BIN-B like spectrum. Further data, or more detailed analysis of historical IceCube data, will be needed to obtain a positive confirmation of the source as a neutrino emitter.

\section{Acknowledgements}

ARdL acknowledges the support of the National Council for Science and Technology from Mexico (CONACYT). AMB and PMC acknowledge the financial support of the UK Science and Technology Facilities Council consolidated grant ST/P000541/1. This work has made use of public Fermi-LAT and IceCube data. Fermipy [16] was used during the development of this project.

\section{References}

[1] M.G. Aarsten et al., [IceCube Collaboration], PRL, Vol. 124, 5 (2020)

[2] M.G. Aarsten et al., [IceCube Collaboration], Astropart. Phys. 92 (2017) 30

[3] M. G. Aartsen et al., [IceCube Collaboration], Science, Vol. 361, no. 6398, 147 (2018)

[4] M. G. Aartsen et al., Science, Vol. 361, no. 6398, eaat1378 (2018)

[5] E. Blaufuss [IceCube Collaboration], GCN, Vol. 28887, no. 1 (2020)

[6] S. Garrappa and S. Buson [Fermi-LAT Collaboration], A. Tel., 14188, 1 (2020)

[7] S. Paiano, A. Tel., 14213, 1 (2020)

[8] S. Buson et al. [Fermi-LAT Collaboration], 14200, 1 (2020)

[9] S. Hümmer et al., APJ, Vol. 721, no. 1, 630 (2010)

[10] Rosales de León et al., MNRAS, Vol. 501, no. 2 (2020)

[11] A. Dominguez et al., MNRAS, 410, 2556 (2011)

[12] Halzen and Hooper, APP, Vol. 23, no. 6, 537 (2005)

[13] G. Ghisellini, MNRAS, Vol. 280, no.1, 67 (1996)

[14] G. Ghisellini, Nature, Vol. 515, 376 (2014)

[15] Bednarz and Ostrowski, PRL, Vol. 80, no. 18, 3911 (1998)

[16] Wood et al. [Fermi-LAT Collaboration], ICRC 2017, Vol. 301, 824 (2017)

[17] S. Abdollahi et al., APJS, Vol. 247, no.1, 33 (2020) 